\title{
Metastability bounds on flavor-violating trilinear soft terms in the MSSM
}

\author{
Jae-hyeon Park \\ Deutsches Elektronen-Synchrotron DESY, Notkestraße 85, 22607 Hamburg, Germany
}

(Received 27 January 2011; published 23 March 2011)

\begin{abstract}
The vacuum stability bounds on flavor-violating trilinear soft terms are revisited from the viewpoint that one should not ban a standard-model-like false vacuum as long as it is long-lived on a cosmological time scale. The vacuum transition rate is evaluated numerically by searching for the bounce configuration. Like stability, a metastability bound does not decouple even if sfermion masses grow. Apart from being more generous than stability, the new bounds are largely independent of Yukawa couplings except for the stop trilinears. With vacuum longevity imposed on otherwise arbitrary $L R$ insertions, it is found that a super flavor factory has the potential to probe sparticle masses up to a few $\mathrm{TeV}$ through $B$ and $\tau$ physics whereas the MEG experiment might cover a far wider range. In the stop sector, metastability is more restrictive than any existing experimental constraint such as from electroweak precision data. Also discussed are dependency on other parameters and reliability under radiative corrections.
\end{abstract}

DOI: 10.1103/PhysRevD.83.055015

PACS numbers: 12.60.Jv, 03.65.Xp, 13.20.He, 13.35.-r

\section{INTRODUCTION}

Flavor physics is an important means to test any theoretical description of elementary particles. Among numerous proposals, the one still most compelling would be the minimal supersymmetric standard model (MSSM) that offers solutions to problems not addressed by the standard model (SM). In the MSSM, the Lagrangian has soft supersymmetry breaking terms. Among them, the scalar mass and the trilinear terms are two representative sources of flavor/CP violation apart from the Cabibbo-KobayashiMaskawa (CKM) matrix. Unless tuned in a particular way, they could add to an existing flavor-changing-neutralcurrent (FCNC) process such as $B \rightarrow X_{s} \gamma$ or create a new one, e.g., $\mu \rightarrow e \gamma$, through loop corrections. In this way, flavor physics provides an invaluable input to the construction of an underlying theory that implements spontaneous supersymmetry or flavor symmetry breaking.

For the same reason, flavor physics has been a traditional tool to unveil supersymmetry. Now that the Large Hadron Collider (LHC) has started its successful operation, the significance of this role has diminished. Nevertheless, there are at least two areas in which flavor studies could make contributions. The first is to scrutinize the flavor structure of newly discovered particles. This is a complement to the LHC that is less sensitive to extra flavor/CP violation. The second is to continue the search for supersymmetric particles. This should be vital particularly in a scenario where sparticle masses are around the LHC reach or beyond. In either case, the crucial quantity is the size of a flavor-violating term in the Lagrangian, often represented by the dimensionless quantity $\delta$ to be defined in the next section.

According to the chirality structure, a mass insertion (MI) can be classified as either chirality-preserving or chirality-flipping. In a high sparticle mass region, the latter type becomes generically more important than the former
[1]. Taking, for example, the ratio of the one-loop $B \rightarrow$ $X_{s} \gamma$ amplitudes caused by the $L R$ and the $L L$ insertions, one finds a quantity proportional to $\left(M_{3} / m_{b}\right) \times$ $\left[\left(\delta_{23}^{d}\right)_{L R} /\left(\delta_{23}^{d}\right)_{L L}\right]$ (see, e.g., Ref. [2]). It shows that the relative contribution from the $L R$ insertion grows linearly as the gluino mass $M_{3}$. This is one of the reasons why the subject of this study is the trilinear terms.

The obvious problem is that one needs to get an idea of the flavor structure of particles that might yet be found. A popular strategy is to employ a model or ansatz that leads to a particular pattern of the soft terms. There are many works in this approach, and this article is not going to be another. The plan here is the following. The most optimistic scenario is presumed in which any $L R$ insertion can take an arbitrary value as long as it stays within existing experimental and theoretical limits. Using this input, one can estimate the maximum possible new signal in an FCNC process. Then it is compared with sensitivities of running or forthcoming experimental searches.

As for the input mentioned above, one would learn nothing new if only the present FCNC limits were used. One needs information from outside flavor physics. There are such theoretical bounds that arise from vacuum stability. If a trilinear term is too large, the MSSM scalar potential develops a charge- and/or color-breaking (CCB) vacuum deeper than the standard-model-like (SML) local minimum [3,4] or an unbounded-from-below (UFB) direction in the field space [4]. Most notably, the conceptual design report of a super flavor factory (SFF) has made an assessment of its ability to reconstruct a $L R$ squark insertion, imposing these bounds [5].

However, it is questionable whether the vacuum stability is an essential requirement, in particular, when one is performing an analysis of generic soft terms that is supposed to be objective. More sensibly, one could use the lifetime of the SML vacuum as the criterion. This viewpoint would not make sense if the Universe were driven 
away from there in its history. In the case of a squark direction, its temperature-dependent mass [6] may defer the formation of a CCB minimum until the electroweak phase transition has happened [7,8]. Supersymmetry breaking by inflation provides a more general mechanism to lift flat directions so that relaxation to the symmetric phase can take place [9]. The bottom line is that there seems to be no definite reason to preclude a lower energy CCB state just for its existence. In this approach, there have been attempts to obtain a viable parameter space of the flavor-conserving stop trilinear $[7,10]$ or the stau trilinear coupling [11], the sparticle [12,13] or the Higgs soft masses [14], or messenger couplings for gauge mediation [15]. This work is an application of the same idea to the flavor-violating trilinear couplings.

The rest of the article begins with an abridged account of theoretical backgrounds in Sec. II. In Sec. III, more practical details of the analysis method are given. The metastability bounds thus obtained are presented in Sec. IV in which their behaviors are also explained. Section V is devoted to an application of the findings to flavor physics. Finally, the article is summarized in Sec. VI.

\section{THEORY}

For concise presentation, the following notations shall be employed. The species of a given matter field is denoted by $f$ which can be one of $e, d, u$, standing for charged leptons, down-, and up-type quarks, respectively. A field symbol without the superscript $c$ refers to a component of the $\mathrm{SU}(2)$ doublets, $L=(\nu, e), Q=(u, d), H_{d}=$ $\left(H_{d}^{0}, H_{d}^{-}\right), H_{u}=\left(H_{u}^{+}, H_{u}^{0}\right)$. Also, another redundant symbol for the down-type Higgs doublet is introduced, i.e., $H_{e} \equiv H_{d}$.

The scalar potential of the MSSM is in the form

$$
V=V_{D}+V_{F}+V_{\text {soft }},
$$

where the $D$-, the $F$-, and the soft term contributions read

$$
\begin{gathered}
V_{D}=\frac{1}{2} \sum_{a} g_{a}^{2}\left(\sum_{\alpha} \phi_{\alpha}^{\dagger} T^{a} \phi_{\alpha}\right)^{2}, \\
V_{F}=\sum_{\alpha}\left|\frac{\partial W}{\partial \phi_{\alpha}}\right|^{2}, \\
V_{\text {soft }}=\tilde{L}_{i}^{\dagger}\left(M_{L}^{2}\right)_{i j} \tilde{L}_{j}+\tilde{e}_{i}^{c \dagger}\left(M_{e^{c}}^{2}\right)_{i j} \tilde{e}_{j}^{c}+\tilde{Q}_{i}^{\dagger}\left(M_{Q}^{2}\right)_{i j} \tilde{Q}_{j} \\
+\tilde{d}_{i}^{c \dagger}\left(M_{d^{c}}^{2}\right)_{i j} \tilde{d}_{j}^{c}+\tilde{u}_{i}^{c \dagger}\left(M_{u^{c}}^{2}{ }_{i j} \tilde{u}_{j}^{c}\right. \\
+2 \operatorname{Re}\left[H_{d} \tilde{L}_{i} A_{i j}^{e} \tilde{e}_{j}^{c}+H_{d} \tilde{Q}_{i} A_{i j}^{d} \tilde{d}_{j}^{c}-H_{u} \tilde{Q}_{i} A_{i j}^{u} \tilde{u}_{j}^{c}\right] \\
+m_{H_{d}}^{2}\left|H_{d}\right|^{2}+m_{H_{u}}^{2}\left|H_{u}\right|^{2}+2 \operatorname{Re}\left[b H_{u} H_{d}\right],
\end{gathered}
$$

with the superpotential

$$
W=H_{d} L_{i} \lambda_{i j}^{e} e_{j}^{c}+H_{d} Q_{i} \lambda_{i j}^{d} d_{j}^{c}-H_{u} Q_{i} \lambda_{i j}^{u} u_{j}^{c}+\mu H_{u} H_{d} .
$$

The index $\alpha$ in (2) and (3) runs over each superfield in the model and $\phi_{\alpha}$ is its scalar component.

For discussion of supersymmetric flavor violation, it is convenient to use the language of mass insertion approximation [16]. In order to specify the families of a given species $f$, one should take the super-CKM basis that leads to the diagonal Yukawa matrix, $\lambda_{i j}^{f}=\lambda_{f_{i}} \delta_{i j}$ with positive eigenvalues. In this article, a $L R$ mass insertion is related with a trilinear coupling by

$$
\begin{gathered}
\left(\delta_{i j}^{f}\right)_{R L}=\frac{A_{j i}^{f}\left\langle H_{f}^{0}\right\rangle}{\left(M_{\mathrm{av}}^{2}\right)_{j i}^{f}}, \\
\left(M_{\mathrm{av}}^{2}\right)_{i j}^{f} \equiv \frac{1}{2}\left[\left(M_{f}^{2}\right)_{i i}+\left(M_{f^{c}}^{2}\right)_{j j}\right] .
\end{gathered}
$$

Note that this definition of average mass is different from that in Ref. [2], i.e., the geometric mean. Nevertheless, this variance does not matter since the soft scalar masses shall be assumed to be nearly degenerate. A trivial but useful identity obeyed by the $L R$ insertions is $\left(\delta_{i j}^{f}\right)_{L R}=\left(\delta_{j i}^{f}\right)_{R L}^{*}$, which follows from the Hermiticity of a sfermion mass matrix.

It should be instructive to review the stability bounds on flavor-violating trilinear couplings [4], as the potential along a CCB direction helps to understand qualitatively many of the properties of the tunnelling bounds. Suppose that the scalar fields take on values such that

$$
\left|H_{f}^{0}\right|=\left|\tilde{f}_{i}\right|=\left|\tilde{f}_{j}^{c}\right|=a,
$$

for one particular set of $f, i$, and $j$, where $i, j=1,2,3$, are family indices with respect to the super-CKM basis. The other fields are all assumed to vanish. Then one can check that $V_{D}=0$ and the lowest potential energy subject to the above condition is

$$
\begin{aligned}
V_{\mathrm{L} . \mathrm{E} .}= & {\left[2\left(M_{\mathrm{av}}^{2}\right)_{i j}^{f}+m_{H_{f}}^{2}+|\mu|^{2}\right] a^{2}-2\left|A_{i j}^{f}\right| a^{3} } \\
& +\left(\lambda_{f_{i}}^{2}+\lambda_{f_{j}}^{2}\right) a^{4} .
\end{aligned}
$$

Because of the cubic term in $a$, a minimum can appear that is deeper than the SML vacuum unless

$$
\left|A_{i j}^{f}\right|^{2}<\lambda_{f_{\max (i, j)}}^{2}\left[2\left(M_{\mathrm{av}}^{2}\right)_{i j}^{f}+m_{H_{f}}^{2}+|\mu|^{2}\right] .
$$

By imposing this inequality, one can avoid a $\mathrm{CCB}$ minimum.

This theoretical bound does not decouple even if sfermions grow heavier. On the contrary, it gets stronger. This property is most easily demonstrated in terms of a $\delta$ parameter. Using (6), one can translate (10) into the form

$$
\left|\left(\delta_{i j}^{f}\right)_{L R}\right|<m_{f_{\max (i, j)}} \frac{\left[2\left(M_{\mathrm{av}}^{2}\right)_{i j}^{f}+m_{H_{f}}^{2}+|\mu|^{2}\right]^{1 / 2}}{\left(M_{\mathrm{av}}^{2}\right)_{i j}^{f}},
$$


where $m_{f_{k}}$ is the mass of fermion $f_{k}$. This bound scales as the inverse power of the average sfermion mass. This behavior is at variance with an FCNC limit on $\left|\left(\delta_{i j}^{f}\right)_{L R}\right|$ that grows as sfermions become heavier. Another point to notice is that the above restriction is independent of $\tan \beta$. As will be shown, this is not the case with a metastability bound.

In addition to the CCB minima, UFB field directions can appear due to large trilinear couplings [4]. If one is worried only about the existence of a deeper point in the field space, both types of constraints should be taken into account. As far as a tunnelling process is concerned, however, a UFB direction is most likely irrelevant for the following reasons. As one traces this direction starting from the SML vacuum, the potential barrier is generically thicker than along a $\mathrm{CCB}$ direction. Moreover, there is a section of path in which $V_{D}$ does not vanish thereby making the barrier much higher as well. Therefore, a tunnelling process prefers a path close to a CCB direction that obeys $D$ flatness all the way.

Even though expressed in a nice analytic form, the role of a CCB condition as a phenomenological constraint is rather unclear. Instead, the lifetime of the SML vacuum shall be required to be long enough. In a semiclassical approximation [17], the decay probability of a metastable vacuum per unit time per unit volume is given by

$$
\Gamma / V=A \exp (-S[\bar{\phi}]),
$$

where $A$ is a prefactor that needs to be guessed, $S$ is the Euclidean action, and $\bar{\phi}$ is its bounce with $O(4)$ symmetry [18]. This symmetry allows one to use a single coordinate, i.e., the radial distance $\rho$, with respect to which the action can be written

$$
S[\phi(\rho)]=2 \pi^{2} \int_{0}^{\infty} d \rho \rho^{3}\left[\left|\frac{d \phi}{d \rho}\right|^{2}+V(\phi)\right] .
$$

Note that the normalization of the kinetic term is that of complex fields, as $\phi$ is intended to be eventually the MSSM scalars. The field configuration $\bar{\phi}(\rho)$ is a stationary point of $S[\phi(\rho)]$ subject to the boundary conditions,

$$
\bar{\phi}(\rho \rightarrow \infty)=\phi_{+}, \quad \frac{d \bar{\phi}}{d \rho}(\rho=0)=0,
$$

where $\phi_{+}$is the false vacuum.

In the context of the MSSM, $\phi$ represents the vector of scalar fields in the model, and $\phi_{+}$is the metastable SML vacuum. One can evaluate the action by replacing $V$ in (13) with the scalar potential (1) plus a constant term such that $V\left(\phi_{+}\right)=0$.

On the other hand, the prefactor $A$ in (12) is practically impossible to compute. Based on dimensional grounds, it is estimated to be the fourth power of the mass scale appearing in the problem. In this work, $A=(100 \mathrm{GeV})^{4}$ shall be used with which one can estimate the smallest acceptable action. The lifetime of the whole observable Universe staying at the SML vacuum is roughly $V /\left(\Gamma t_{0}^{3}\right)$ where $t_{0} \approx 10 \mathrm{Gyr}$ is the age of the Uuniverse. One can keep this longer than $t_{0}$ by demanding that [10]

$$
S>400 .
$$

The above value of $A$ may be regarded as a little small in view of the sfermion masses in the $\mathrm{TeV}$ range. Choosing a small $A$ implies a conservative parameter space exclusion. Nonetheless, the constraint is much less sensitive to the variation of $A$ than of $S$. If desired, one could translate the multiplicative uncertainty of $A$ into the additive one of $S$ by taking the logarithm. For instance, taking $A$ instead to be the fourth power of the reduced Planck scale would push up the lower bound on $S$ by 150 .

Another type of uncertainty arises from radiative corrections. It is important to estimate their effects especially because the numerical analysis uses the tree-level potential. One can implement the one-loop correction to the tunnelling rate simply by using the one-loop effective potential

$$
V_{1 \mathrm{~L}}=V+\frac{1}{16 \pi^{2}} V^{(1)}+\Omega,
$$

instead of the tree-level potential $V$ in (13). The constant $\Omega$ should be adjusted so that $V_{1 \mathrm{~L}}\left(\phi_{+}\right)=0$. The one-loop correction in the $\overline{\mathrm{DR}}^{\prime}$ scheme is [19]

$$
V^{(1)}=\sum_{n}(-1)^{2 s_{n}}\left(2 s_{n}+1\right) \frac{m_{n}^{4}}{4}\left(\ln \frac{m_{n}^{2}}{Q^{2}}-\frac{3}{2}\right),
$$

where $m_{n}^{2}$ is the field-dependent mass-squared eigenvalue of the $n$-th degree of freedom with spin $s_{n}$. The renormalization scale $Q$ should be chosen so that it minimizes $V^{(1)}$. This means that the optimal scale is a function of $\phi$. Taking this scale would be satisfying but technically involved. For a numerical computation, the scale shall be set equal to the common diagonal component of the soft sfermion masses. This is to be supplemented with error estimation. Fortunately, the bounce consists of $\phi$ limited within a range not too far from the sfermion mass scale [7]. Therefore, $V^{(1)}$ does not diverge even if the CCB minimum is taken to infinity.

One could estimate the uncertainty due to ignoring loop corrections to the action by evaluating the shift made by one-loop,

$$
S_{1 \mathrm{~L}}\left[\bar{\phi}_{1 \mathrm{~L}}\right]-S[\bar{\phi}],
$$

where $S_{1 \mathrm{~L}}$ is given by (13) with $V$ replaced by the real part of the one-loop effective potential $V_{1 \mathrm{~L}}$ in (16) [20], and the functional argument of each action is its bounce. One can approximate the difference (18) by

$$
\Delta S \equiv S_{1 \mathrm{~L}}[\bar{\phi}]-S[\bar{\phi}]=2 \pi^{2} \int_{0}^{\infty} d \rho \rho^{3} \operatorname{Re}[\Delta V(\bar{\phi}(\rho))]
$$

where 


$$
\Delta V=V_{1 \mathrm{~L}}-V,
$$

keeping only the leading order term in $\Delta V$. Note that $\Delta V$ is not simply equal to $V^{(1)} /\left(16 \pi^{2}\right)+\Omega$ as may be suggested by (16) since the parameters entering $V$ and $V_{1 \mathrm{~L}}$ in (20) have different values. In particular, those parameters pertinent to the electroweak symmetry breaking must deviate. Otherwise it would be impossible for both $V$ and $V_{1 \mathrm{~L}}$ to result in realistic Higgs vacuum expectation values.

\section{PROCEDURE OF ANALYSIS}

The method of numerical analysis is spelled out in detail. The same procedure is repeated for each of the species $f=e, d, u$ and for each of the six flavor-off-diagonal trilinear couplings.

The families of the scalars $\tilde{f}$ and $\tilde{f}^{c}$ are defined in the super-CKM basis where the corresponding Yukawa matrix $\lambda_{i j}^{f}$ is diagonal. The CKM mixing is neglected so that both $\lambda^{u}$ and $\lambda^{d}$ are diagonal at the same time. This simplifies the computation as the flavor-mixing Yukawa couplings are dropped from the superpotential. They are of $\mathcal{O}\left(V_{t s} \lambda_{t}\right) \sim \lambda^{2}$ or less, and presumably are not very important in view of the fact that a coupling of similar order $\lambda_{b} \sim 0.1$ does not make a sizeable difference as will be demonstrated later in this article.

As the focus is on flavor violation by the trilinear couplings, the soft masses shall be assumed to be in a universal form,

$$
\left(M_{f}^{2}\right)_{i j}=\left(M_{f^{c}}^{2}\right)_{i j}=m_{\tilde{f}}^{2} \delta_{i j} .
$$

Among the trilinear couplings, one $A_{i j}^{f}$ with $i \neq j$ is scanned with the step size of $0.1 \mathrm{TeV}$ while all the other components are kept zero. The diagonal, of no interest, is set to zero. One might well imagine a situation where more trilinears are turned on in addition to $A_{i j}^{f}$. The additional couplings do not block an existing tunnelling path but may open more [21]. In this sense, the bounds obtained in this work should be regarded as generous.

The phase of a single $A_{i j}^{e}$ can be rotated away by a lepton-flavor number transformation. Similarly, the phase of $A_{i j}^{d}$ or $A_{i j}^{u}$ may be absorbed into the squark fields by a "quark-family number" transformation in the limit of no CKM mixing that is taken here.

The gauge and the Yukawa couplings are set at the renormalization scale $Q=m_{\tilde{f}}$. Therefore, $A_{i j}^{f}$ presented later are also supposed to be at this scale. The other parameters shall be set as follows unless stated otherwise:

$$
\begin{aligned}
\tan \beta & =10, & \mu & =0.5 \mathrm{TeV}, \\
m_{H^{0}} & =0.5 \mathrm{TeV}, & m_{\tilde{q}} & =m_{\tilde{l}}=3 \mathrm{TeV} .
\end{aligned}
$$

The Higgs mass parameters, $m_{H_{d}}^{2}, m_{H_{u}}^{2}$, and $b$, are determined from these by the tree-level electroweak symmetry breaking condition. More parameter space is also explored by varying each of the above. A remark is in order regarding the sign of $\mu$. The only non-negligible term affected by this in the scalar potential is $2 \operatorname{Re}\left[\mu H_{d} \tilde{Q}_{3}^{*} \lambda_{t} \tilde{u}_{3}^{* *}\right]$. Therefore, negating $\mu$ can be compensated for by flipping the sign of either $\tilde{Q}_{3}$ or $\tilde{u}_{3}^{c}$. Note that this can be done without touching the trilinear term in question. In the case of a flavorconserving trilinear, by contrast, the signs of $A_{33}^{u}$ and $\mu$ cannot be disentangled [21].

For faster computation, every scalar field is constrained to be real valued. This is not completely general in that for a given set of real-valued $\left\{\phi_{\alpha}\right\}$,

$$
\min _{0 \leq \theta_{\alpha}<2 \pi} V\left(e^{i \theta_{\alpha}} \phi_{\alpha}\right) \leq \min _{\theta_{\alpha}=0, \pi} V\left(e^{i \theta_{\alpha}} \phi_{\alpha}\right),
$$

even if all the parameters in $V$ are real. One may worry that there can be a complex-valued path in the field space that costs less potential energy. Nevertheless, it can be checked that the equality holds as long as $\left\{\phi_{\alpha}\right\}$ stays sufficiently close to one of the directions in (8), and thus the potential is dominated by a few terms including the activated trilinear term, in particular. It turns out that the numerical bounce does reveal this property.

Under the same condition, one can show that it costs less potential energy for the squark color directions to be aligned. This justifies retaining only one color component of each squark assuming that the others are all zero.

As the problem is concerned with a flavor-off-diagonal coupling, one should put at least two families of scalar fields into action as variable degrees of freedom in addition to the Higgs doublets. For the lepton sector, the following 10 variables are used: $H_{d}, H_{u}, \tilde{L}_{i}, \tilde{e}_{i}^{c}$, where the index $i$ runs over the two families involved. Right-handed sneutrinos are not taken into account. The quark sector needs two more variables: $H_{d}, H_{u}, \tilde{Q}_{i}, \tilde{d}_{i}^{c}, \tilde{u}_{i}^{c}$.

The bounce is computed numerically. The Euclidean action (13) is approximated by a function of the fields that are put on the discretized $\rho$ axis. Each lattice point has an independent set of the aforementioned 10 or 12 degrees of freedom. One can find a stationary point constrained by the boundary conditions (14) using a method derived from that in Ref [22]. The original series of steps had to be modified since it is not suitable for a problem in which the true vacuum is very far from the false vacuum in comparison to the thickness of the potential barrier. This happens if the Yukawa couplings in (9) are small which is mostly the case except for $\lambda_{t}$. The details of the revision are presented in a separate article [23]. With this method, one does not have to know where the true minimum is, or even whether or not the potential is bounded from below. The price to pay is that one needs to minimize a function obeying constraints given in implicit forms. This can be done for instance by using the IPOPT package [24].

At the first stage of the computation, one needs to choose an initial field configuration. The above method needs a trajectory in the field space that connects $\phi_{+}$to any point 
$\phi_{e}$ over the barrier such that $V\left(\phi_{e}\right)=V\left(\phi_{+}\right)$. To find a $\phi_{e}$, a point leaves the SML vacuum and hikes along a valley in the direction of increasing $|\phi|$ until it goes down and touches the initial altitude.

Finally, the SML vacuum lifetime is compared with the age of the Universe using (15) to accept or reject the given parameter set.

Apart from the scanning procedure described above, error estimation is performed for a few selected cases by evaluating the approximate one-loop effect (19). The parameters entering the effective potential (16) are generated by SOFTSUSY [25] with options set for one-loop Higgs masses and tadpoles. ${ }^{1}$ Unlike the tree-level potential, $V_{1 \mathrm{~L}}$ depends on the gaugino masses as well. They are picked up so that $M_{1,2,3}=m_{\tilde{f}}$ for order-of-magnitude estimation.

\section{UPPER BOUNDS AND INTERPRETATIONS}

Requiring that the bounce action obeys the condition (15), one gets the upper bounds on the off-diagonal trilinear couplings shown in Fig. 1. It turns out that the six components of $A_{i j}^{e}$ have almost the same bounds (falling within $100 \mathrm{GeV}$ from one another), and therefore a single vertical line is used to display them all together. The same is true of the six components of $A_{i j}^{d}$. As for $A_{i j}^{u}$, the upper bounds highly depend on whether either of the family indices is 3 or not. The bounds on $A_{12}^{u}$ and $A_{21}^{u}$ can be displayed together, and likewise the other four couplings.

One can notice several features on the plot. First of all, the upper bound on an off-diagonal $A$ grows as the corresponding sfermion mass is increased. The dependence turns out to be almost linear although not exactly. Second, the bound is symmetric under the interchange of the two family indices. Third, the bound on $A_{i j}^{e}$ is independent of $i$ and $j$ as mentioned above. This family independence is discovered also in $A_{i j}^{d}$. This property contrasts with the Yukawa dependence of the stability bounds (10). Fourth, the bound on $A_{i j}^{e}$ is nearly the same as that on $A_{i j}^{d}$. Fifth, the bound on $A_{12}^{u}$ is slightly lower than that on $A_{i j}^{e, d}$. Sixth, the bound on $A_{13,23}^{u}$ is higher than that on $A_{12}^{u}$ and also tend to be higher than the other bounds for $m_{\tilde{f}} \gtrsim 1 \mathrm{TeV}$. Interpretations of these observations shall be given in what follows.

For understanding the results, it should be instructive to examine contributions from different components of the scalar potential separately. Three instances are shown in Fig. 2, one with lepton flavor violation (LFV) and the other two with up-type quark flavor change. The figure for the down-quark sector is nearly identical to Fig. 2(a) and therefore has been omitted. Each plot displays the differential action that arises from each of $V_{D}, V_{F}, V_{\text {soft }}$, and their

\footnotetext{
${ }^{1}$ There is a bug in SOFTSUSY version 3.1.7 that discards even one-loop tadpoles when the option numRewsbLoops is set to 1 . This problem does not appear with the default setting.
}

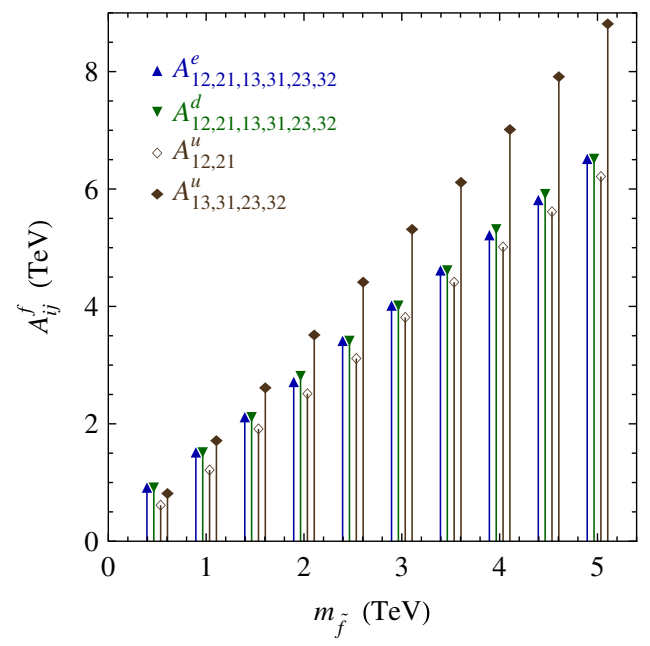

FIG. 1 (color online). Upper bounds on flavor-violating $A_{i j}^{f}$ as functions of $m_{\tilde{f}}$ for $f=e, d, u$ and $i, j=1,2,3$. They correspond to the following choice of parameters: $\tan \beta=10$, $\mu=0.5 \mathrm{TeV}, m_{H^{0}}=0.5 \mathrm{TeV}$. Each group of four vertical lines shares the same value of $m_{\tilde{f}}$ given by the average of their horizontal coordinates.

sum denoted by $V$, as one moves along the trajectory of the bounce profile. The horizontal axis is the distance traversed in the field space starting from the point of $\rho=0$. Every curve arrives on the right at the false vacuum. Therefore, the area under (or over) each curve is the action coming from the given potential component. Also shown is the (positive-definite) differential action from the kinetic term. Obviously, the total Euclidean action is given by the sum of the area under the light grey curve and the area of the light grey (yellow) region. The parameters used in Fig. 2 are as in (22) besides the trilinear couplings indicated in the caption. Each parameter set leads to the action $S \approx 400$, that is, the $A$ coupling is around the metastability limit.

On each plot, the area under the $V_{D}$ curve is tiny compared to that under $V$. This means that $V_{D}$ does not play a very important role in determining the action among the different components of the total scalar potential $V$. This point appears more concrete in Table I which lists the numerical value of each area. Examining the field configuration, one can indeed find that the trajectory of the solution almost follows one of the $D$-flat directions in (8). The same holds in every case considered in this work in either the lepton or the quark sector. This allows one to understand the qualitative features of the metastability bound on a trilinear coupling by looking at the potential (9). One property of $V_{\text {L.E. }}$ is that it is symmetric under the interchange of $i$ and $j$ if both $\left(M_{\mathrm{av}}^{2}\right)_{i j}^{f}$ and $A_{i j}^{f}$ are symmetric. This makes it understandable that the bound on $A_{i j}^{f}$ is symmetric. However, this apparent symmetry stems from the assumed form of the sfermion mass matrix in (21). Nevertheless, one could make a straightforward guess at the behavior for a more general mass matrix: the bound 

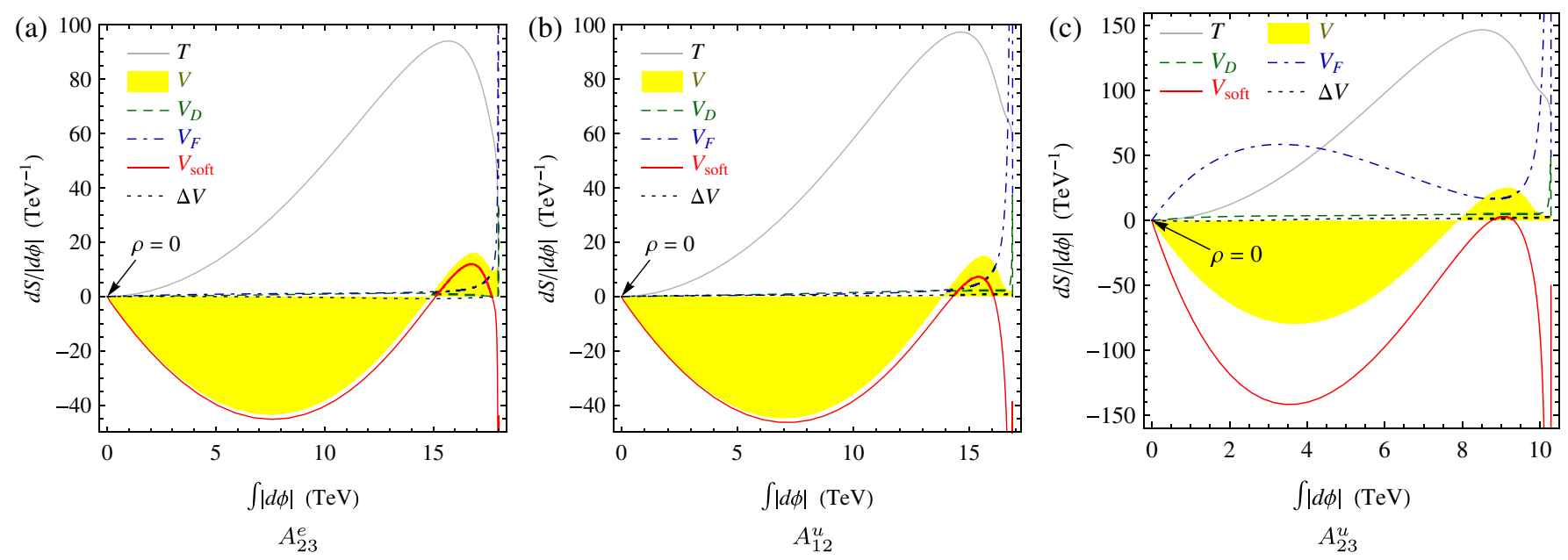

FIG. 2 (color online). Contributions of different potential components to the Euclidean bounce action. The nonzero trilinear coupling is (a) $A_{23}^{e}=4 \mathrm{TeV}$, (b) $A_{12}^{u}=3.8 \mathrm{TeV}$, or (c) $A_{23}^{u}=5.3 \mathrm{TeV}$. The (signed) light grey (yellow) areas display the action coming from the total potential energy density $V$ that is the sum of $V_{D}, V_{F}$, and $V_{\text {soft }}$, represented by the dashed (green), the dot-dashed (blue), and the solid grey (red) curves, respectively. A black dotted curve is the estimated one-loop correction. The kinetic term is also displayed as a light grey curve.

should be a function of $\left(M_{\mathrm{av}}^{2}\right)_{i j}^{f}$. This property has been verified numerically.

The second smallest contribution comes from $V_{F}$. It is totally negligible in a case for $A_{i j}^{e}$ or $A_{i j}^{d}$. This explains why the upper bounds on $A_{i j}^{e}$ and $A_{i j}^{d}$ nearly coincide in Fig. 1. They do not depend on $i$ or $j$, and are even independent of whether they are for charged sleptons or down-type squarks. The only differences between the two species are their gauge couplings provided that their masses and trilinear couplings are equal and that their Yukawa couplings are ignored. Since both $S_{F}$ and $S_{D}$ are negligible, the Yukawa and the gauge couplings do not make a noticeable difference between the bounds on $A_{i j}^{e}$ and $A_{i j}^{d}$. This should be clear from the potential in (9) with the Yukawa terms discarded. As for $A_{12}^{u}, S_{F}$ is not as small but still minor compared to $S_{\text {soft }}$. Its bound in Fig. 1 is quite close to but a little stronger than those on $A_{i j}^{e, d}$, although the charm Yukawa is small as well. This split stems from the fact

TABLE I. The Euclidean action $S$ decomposed into parts coming from the kinetic term, $V_{D}, V_{F}, V_{\text {soft }}$, and $V$, respectively, labeled $S_{T}, S_{D}, S_{F}, S_{\text {soft }}$, and $S_{V}$, in each of the three cases shown in Fig. 2. The last row shows the estimated one-loop effect.

\begin{tabular}{lrrr}
\hline \hline Panel in Fig. 2 & (a) $A_{23}^{e}$ & (b) $A_{12}^{u}$ & (c) $A_{23}^{u}$ \\
\hline$S_{T}$ & 781 & 763 & 749 \\
$S_{D}$ & 12 & 22 & 41 \\
$S_{F}$ & 39 & 116 & 529 \\
$S_{\text {soft }}$ & -446 & -524 & -947 \\
$S_{V}$ & -395 & -385 & -377 \\
$S$ & 386 & 378 & 372 \\
$\Delta S$ & -6 & 8 & 11 \\
\hline \hline
\end{tabular}

that $A_{12}^{u}$ is associated with the up-type Higgs unlike $A_{i j}^{e, d}$. This point shall be elaborated on later.

A trilinear coupling including a stop is allowed to be substantially larger than $A_{12}^{u}$ even though they involve the same Higgs field, as can be found in Fig. 1. The reason is the large top Yukawa coupling. It is evident in (9) that Yukawas lift the scalar potential as quartic couplings, and so they slow down the tunnelling process if they are large. Therefore, large $\lambda_{t}$ implies weaker restrictions on $A_{13,23}^{u}$. The nontrivial role of $\lambda_{t}$ manifests itself in both Fig. 2(c) and the rightmost column of Table I which show that $V_{F}$ is not negligible as for the other $A$ terms.

The last element to be explained in Table I is the estimate of one-loop effects, which was defined in (19). The differential version is plotted in Fig. 2. The magnitude of $\Delta S$ turns out to be roughly $3 \%$ of the tree-level $S$ in the case of $A_{23}^{u}$ and is less significant in the other two. By full computation of the one-loop bounce $\bar{\phi}_{1 \mathrm{~L}}$, the validity of $\Delta S$ as an approximation of the one-loop shift in (18) has also been checked. The latter quantity is indeed of the same order as $\Delta S$ for any case shown in the table. In combination with the smallness of $\Delta S$, this should a posteriori support the reliability of an analysis employing the tree-level effective potential.

Nevertheless, one should like to translate the variation in $S$ into that in the maximum $A_{i j}^{f}$. In any case, there are nonvanishing uncertainties coming from loop corrections as well as from the ignorance of the prefactor. Also, one could choose to impose a lower limit on $S$ slightly different from that in (15). All these variances could be encoded as a constant added to $S$. Then, its influence on a trilinear coupling can be found in Fig. 3. If one allows for a change in $S$ by a factor between 0.6 and 1.6 for instance, the bound 

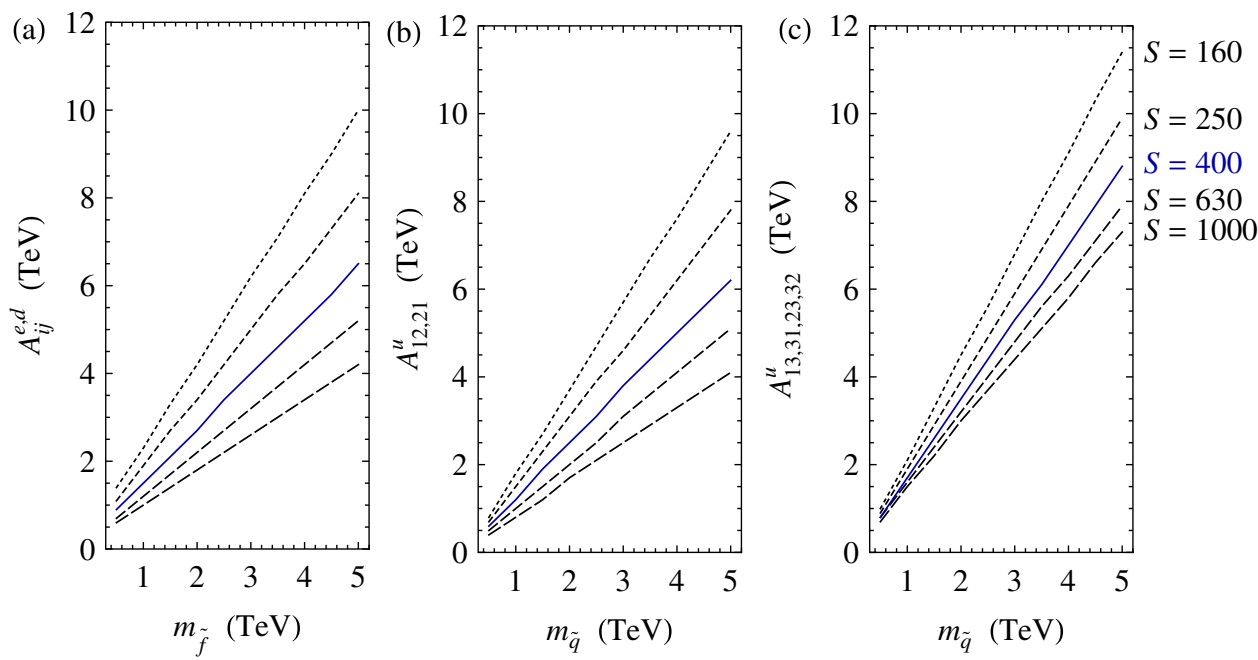

FIG. 3 (color online). The plots show how the upper bounds change depending on the condition imposed on $S$. On each curve, the value of $A_{i j}^{f}$ leads to the corresponding $S$ shown on the right, where $f=e, d, u$ and $i, j=1,2,3$. The following values of parameters were used: $\tan \beta=10, \mu=0.5 \mathrm{TeV}, m_{H^{0}}=0.5 \mathrm{TeV}$.

on $A_{i j}^{f}$ is altered by ${ }_{-20}^{+30} \%$, and each metastability bound presented later is weakened or strengthened by the same fraction.

As is clear in Fig. 1, the tunnelling process gets slower as the squark and the slepton masses increase since they lift the potential (9) quadratically. By the same token, one may envisage a similar effect from higher masses of Higgs fields, as either of them also acquires a vacuum expectation value along the CCB direction (8). Concerning the Higgs bosons, the parameters directly linked to the tunnelling are not the soft masses appearing in (9) (which can be tachyonic) but the physical mass eigenvalues which describe the positive curvature of the potential around the SML vacuum. In the MSSM, one can specify the tree-level Higgs potential by choosing $\tan \beta, m_{H^{0}}$, and $\mu$. Once the former two parameters fix the complete Higgs mass spectrum, varying $\mu$ makes no difference therein. The dependence on each of these three parameters shall be discussed below.

First, $m_{H^{0}}$ and the sfermion masses are varied while the other parameters are fixed according to (22). The results are set out in Figs. 4 as grey contours. As expected, enlarging $m_{H^{0}}$ does provide more room for the trilinear couplings. However, the effect is significant only on those involving the down-type Higgs, displayed in Fig. 4(a). Contours of maximum $A_{i j}^{u}$ in Figs. 4(b) and 4(c), do not exhibit a noticeable dependence on $m_{H^{0}}$. One can understand this by considering Higgs mixing. The real part of each Higgs field can be expressed as (see, e.g., Ref. [26])

$$
\begin{aligned}
& \operatorname{Re} H_{u}^{0} \approx v_{u}+\left(\sin \beta h^{0}-\cos \beta H^{0}\right) / \sqrt{2}, \\
& \operatorname{Re} H_{d}^{0} \approx v_{d}+\left(\sin \beta H^{0}+\cos \beta h^{0}\right) / \sqrt{2},
\end{aligned}
$$
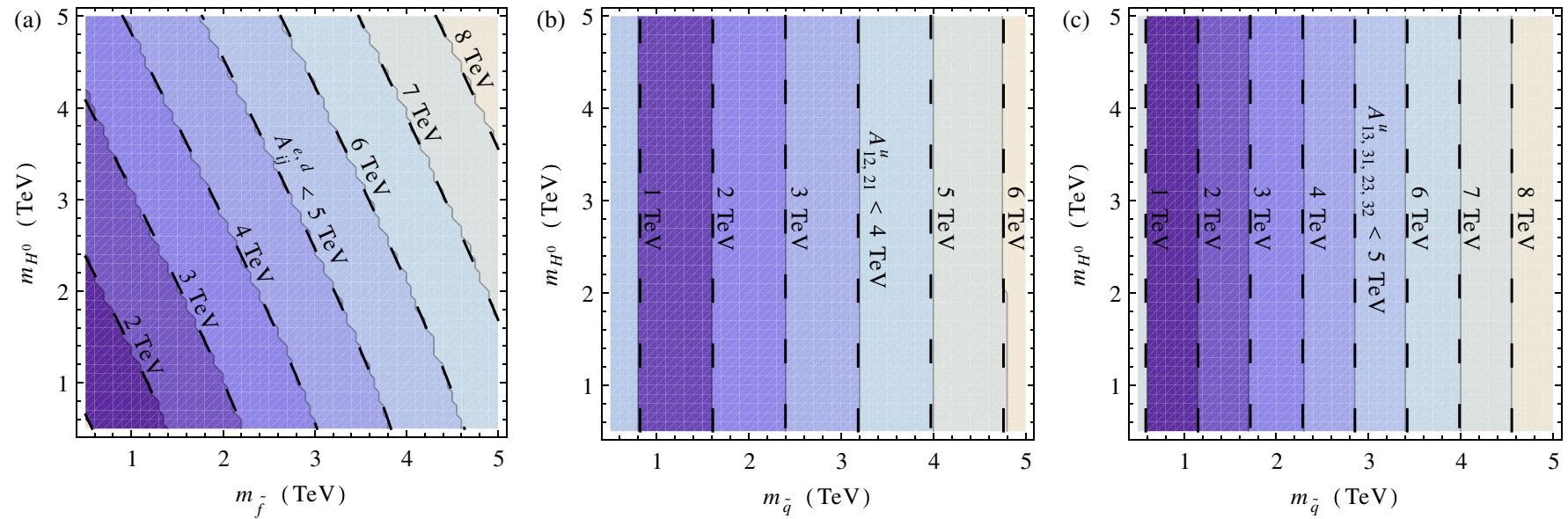

FIG. 4 (color online). Contours of upper bounds on flavor-violating $A_{i j}^{f}$ on the $\left(m_{\tilde{f}}, m_{H^{0}}\right)$ plane for $f=e, d, u$ and $i, j=1,2,3$. The grey contours [which look wiggly in Fig. 4(a)] are based on the data from grid scanning and the dashed black curves are the leastsquares fits. They correspond to the following choice of parameters: $\tan \beta=10, \mu=0.5 \mathrm{TeV}$. 

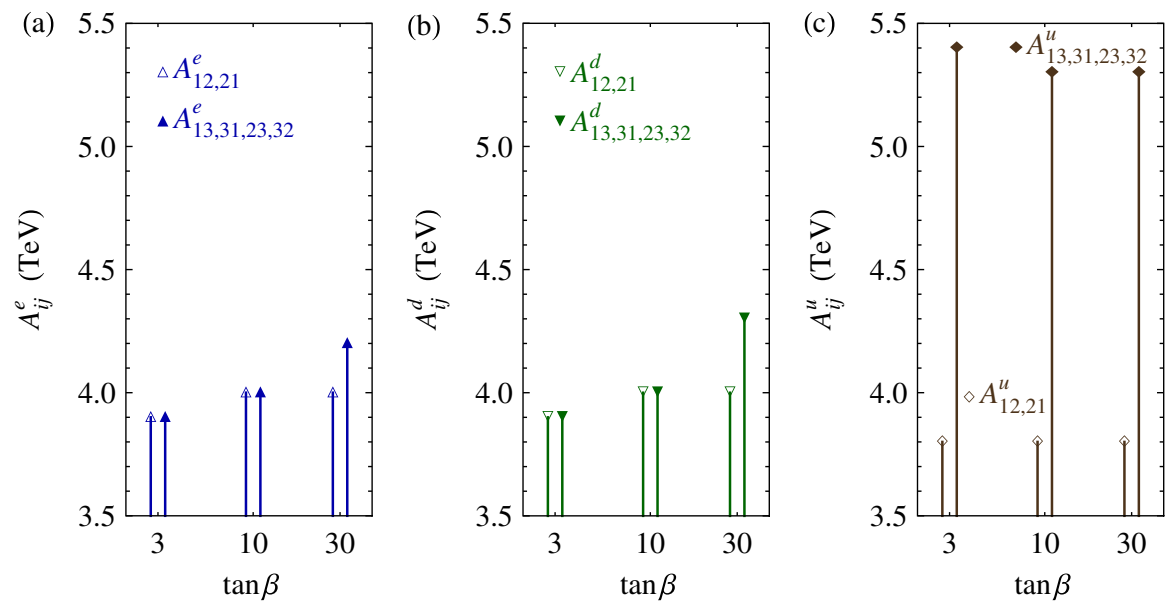

FIG. 5 (color online). Upper bounds on flavor-violating $A_{i j}^{f}$ as functions of $\tan \beta$ for $f=e, d, u$, and $i, j=1,2,3$. They correspond to the following choice of parameters: $\mu=0.5 \mathrm{TeV}, m_{H^{0}}=0.5 \mathrm{TeV}, m_{\tilde{f}}=3 \mathrm{TeV}$. Each pair of vertical lines shares the same $\tan \beta$.

in terms of the mass eigenstates. The Higgs mixing angle $\alpha$ has been approximated by $\beta-\pi / 2$ since $m_{H^{0}} \gg m_{Z}$ in most of the parameter space considered in this work. For $\tan \beta=10, H_{u}^{0}$ and $H_{d}^{0}$ are mostly $h^{0}$ and $H^{0}$, respectively. Since the mass of the lighter $C P$-even neutral Higgs boson is always restricted around $m_{Z}$, the potential curvature along the $H_{u}^{0}$ direction stays essentially constant in comparison to the large variation in the squark mass. This property should remain true at least qualitatively for any $\tan \beta$ bigger than a few.

A metastability bound should be easier to use if one has its algebraic expression. Within the parameter region shown in the figures, the bounds can be approximated by the empirical inequalities,

$$
\begin{aligned}
A_{i j}^{e, d}< & 1.61 m_{\tilde{f}}+0.59 m_{H^{0}}-0.45 m_{\tilde{f}}^{0.93} m_{H^{0}}^{0.07} \\
& +37 \mathrm{GeV}, \\
A_{12,21}^{u}< & 1.27 m_{\tilde{q}}-44 \mathrm{GeV}, \\
A_{13,31,23,32}^{u}< & 1.76 m_{\tilde{q}}-32 \mathrm{GeV} .
\end{aligned}
$$

These are the least-squares fits to the numerical data using the form $a m_{\tilde{f}}+b m_{H^{0}}+c m_{\tilde{f}}^{p} m_{H^{0}}^{1-p}+d$. They are plotted as dashed black curves in Fig. 4, which reveals that the fits are indeed faithful approximations of the data. These inequalities should remain nearly the same even for different values of $\tan \beta$ and $\mu$ since the bounds are insensitive to them as will be shown below. It should also be remembered that $m_{\tilde{f}}^{2}$ should be replaced by $\left(M_{\mathrm{av}}^{2}\right)_{i j}^{f}$ if the diagonal soft masses are highly nondegenerate.

Next, the dependence on $\tan \beta$ is illustrated in Fig. 5. There are two origins of change as $\tan \beta$ varies. One is the Higgs mixing mentioned above. This works in the same way on all the species that couple to each type of Higgs. As $\tan \beta$ increases, $H_{d}^{0}$ in (24b) acquires more fraction of the heavier Higgs which impedes the tunnelling caused by $A_{i j}^{e, d}$. This effect appears as the slight rise of the $A_{12}^{e, d}$ points for higher $\tan \beta$ in Figs. 5(a) and 5(b). For $A_{i j}^{u}$, the change is in the opposite way but too small to be visible on the points for $A_{12}^{u}$ in Fig. 5(c). Remember that increasing $m_{H^{0}}$ renders the tunnelling less probable. Therefore, the influence of Higgs mixing should be enhanced by higher $m_{H^{0}}$. The other origin is the variation of Yukawa couplings, which dominates the $\tan \beta$ dependence of the trilinear couplings involving the third family. A higher value of $\tan \beta$ implies larger down-type quark and charged lepton Yukawas. This gives rise to slower tunnelling as explained before. This splits to some extent the upper bounds that were close together for lower $\tan \beta$. The up-type Yukawas do not change as much as the down-type ones, and this leads to the milder dependence of $A_{13,23}^{u}$ in Fig. 5(c). After all, the alteration is at most $10 \%$, found in $A_{23}^{d}$, as $\tan \beta$ is increased from 3 to 30, which does not seem very significant.

Finally, one should discuss the dependency on $\mu$. As already stated, the Higgs mass eigenvalues and mixing angle do not rely on $\mu$ after $\tan \beta$ and $m_{H^{0}}$ have been fixed. Therefore, the metastability bounds should remain the same while $\mu$ is altered. This has been checked by trying two values of $\mu$ of different orders of magnitude: $0.5 \mathrm{TeV}$ and $3.0 \mathrm{TeV}$. For each $\mu, m_{H^{0}}$ has been scanned from 0.5 TeV to 5.0 TeV, with the other parameters set as (22). At these parameter points, the two choices of $\mu$ do lead to identical upper bounds.

\section{IMPLICATIONS FOR FLAVOR PHYSICS}

Having obtained the limits on flavor-violating trilinear couplings, one should consider what consequences they have for physical processes. In what follows, the metastability bounds shall be contrasted with other existing constraints. They are based primarily on new physics 
TABLE II. Present and future experimental sensitivities to the branching ratios of the lepton flavor-violating decay modes that probe $\left(\delta_{i j}^{e}\right)_{L R}$.

\begin{tabular}{lcc}
\hline \hline Mode & Current bound & Future bound \\
\hline$\mu \rightarrow e \gamma$ & $1.2 \times 10^{-11}[27]$ & $1 \times 10^{-13}[28]$ \\
$\tau \rightarrow e \gamma$ & $3.3 \times 10^{-8}[29]$ & $2 \times 10^{-9}[5]$ \\
$\tau \rightarrow \mu \gamma$ & $4.4 \times 10^{-8}[29]$ & $2 \times 10^{-9}[5]$ \\
\hline \hline
\end{tabular}

searches within FCNC processes, of which prospects at running and planned experiments are also discussed. Other indirect bounds are compared together such as those coming from the vacuum stability, the $\rho$ parameter, and naturalness of the CKM matrix. In this section, the degree of flavor-violation is expressed as a $\delta$ parameter, defined in (6), that is normally used for flavor physics.

The strongest experimental constraints on the $L R$ mass insertions in the slepton sector come from radiative LFV decays. The restrictions on their branching ratios are collected in Table II. The current upper limits are all at $90 \%$ confidence level (C.L.). The quoted future sensitivity to $\mu \rightarrow e \gamma$ is anticipated at $90 \%$ C.L. from the MEG experiment [28]. According to Ref. [5], a SFF is expected to provide the displayed $90 \%$ C.L. bounds on $\tau \rightarrow e \gamma$ and $\tau \rightarrow \mu \gamma$, whereas Ref. [30] projects the $\tau \rightarrow \mu \gamma$ bound of $3 \times 10^{-9}$

Each bound is translated into that on the corresponding mass insertion and presented below. For this, one should choose the other parameters on which the LFV decay rate depends. In the case of $\left(\delta_{i j}^{e}\right)_{L R}$ or $\left(\delta_{i j}^{e}\right)_{R L}$ with $j>i$, fixing the bino mass $M_{1}$ is enough to determine $B\left(l_{j} \rightarrow l_{i} \gamma\right)$ in the mass insertion approximation [31]. As a demonstration, it shall be assumed that $M_{1}=m_{\tilde{l}}$.

In the squark sector, there are multiple observables that restrict a single mass insertion. These constraints are combined to give the $95 \%$ C.L. upper bound on the modulus of each mass insertion in Table III. Also presented are the moduli that in the future can be reconstructed at the level of $3 \sigma$. They are based on the estimates in Ref. [5]. An estimate in this reference is in the form of a range and the values in Table III are those at the lower ends, i.e., the most optimistic ones. The limit depends on the squark and the gluino masses as it arises from gluino-squark loops.

TABLE III. Experimental sensitivities to flavor-violating mass insertions in the squark sector for $m_{\tilde{q}}=M_{3}=1 \mathrm{TeV}$. Expectations of future searches are quoted if available.

\begin{tabular}{lcc}
\hline \hline MI & Current bound & Future bound \\
\hline$\left(\delta_{12}^{d}\right)_{L R, R L}$ & $1.4 \times 10^{-3}[32]$ & \\
$\left(\delta_{13}^{d}\right)_{L R, R L}$ & $2.9 \times 10^{-2}[32,33]$ & $2 \times 10^{-3}[5]$ \\
$\left(\delta_{23}^{d}\right)_{L R, R L}$ & $1.4 \times 10^{-2}[32]$ & $5 \times 10^{-3}[5]$ \\
$\left(\delta_{12}^{u}\right)_{L R, R L}$ & $1.6 \times 10^{-2}[32]$ & \\
\hline \hline
\end{tabular}

The numerical values shown in the table are for $m_{\tilde{q}}=$ $M_{3}=1 \mathrm{TeV}$, and they are proportional to the squark mass as long as $m_{\tilde{q}} / M_{3}$ is fixed. This ratio shall be maintained in the following plots.

The FCNC constraints presented below should be regarded as rough estimates. They rely on parameters that do not affect a metastability bound. Already at one-loop level, they depend on gaugino masses which have been chosen somewhat arbitrarily as stated above. Moreover, chirally enhanced higher order corrections can significantly strengthen or weaken a flavor constraint [34]. These corrections shall be ignored that depend on yet more parameters.

Given the aforementioned experimental information, one could start the comparative analysis by examining the charged leptons. In Fig. 6, the vacuum bounds are displayed along with the present and the projected sensitivities of FCNC searches. The vacuum stability border is given by (11), above which the corresponding trilinear term gives rise to tunnelling. If one abandons the requirement that the SML vacuum be absolutely stable, then the limit is relaxed up to the line above which the false vacuum decays too fast in comparison to the age of the Universe. As the sfermion masses increase, both the stability and the metastability bounds become stronger. However, the latter depends on $\tan \beta$ unlike the former. As Fig. 5(a) shows, maximum $A_{i j}^{e}$ allowed by the vacuum lifetime is insensitive to $\tan \beta$. Therefore, (6) implies a tighter restriction on $\left(\delta_{i j}^{e}\right)_{L R}$ for higher $\tan \beta$. In contrast, the FCNC constraints loosen as the sfermions grow heavier.

More specifically, Fig. 6(a) is concerned with the $\mu \rightarrow e$ transition. Within the displayed range, the tunnelling process does not play a significant role in comparison to the $\mu \rightarrow e \gamma$ decay. The MEGA experiment has ruled out more region than metastability so long as $m_{\tilde{l}} \lesssim 20 \mathrm{TeV}$ for $\tan \beta=30$. There is even no probability of tunnelling for up to $m_{\tilde{l}} \approx 3 \mathrm{TeV}$, beyond which the SML vacuum lifetime is still permitted to be either finite or infinite. Allowing for an arbitrary mass insertion consistent with each vacuum bound, one can estimate what slepton mass range may be accessible through $\mu \rightarrow e \gamma$. The stability condition sets the higher end around $10 \mathrm{TeV}$. If one opens up the possibility of a long-lived false vacuum, the territory extends up to $m_{\tilde{l}} \sim 60 \mathrm{TeV}$ where the metastability bound for $\tan \beta=30$ crosses the MEG sensitivity. As is clear in the plot, this crossing point depends on $\tan \beta$, and is about $100,200 \mathrm{TeV}$ for $\tan \beta=10,3$, respectively. Above this mass scale, the mass insertion is suppressed too much to be observed. The metastability bound has been numerically computed only up to $m_{\tilde{l}}=5 \mathrm{TeV}$, and the preceding estimates are based on linear extrapolation.

In a similar way, one can interpret Fig. 6(b) which shows the 1-3 and the 2-3 mixings at the same time. Obviously, they are associated with $\tau \rightarrow e \gamma$ and $\tau \rightarrow \mu \gamma$, respectively. In contrast to $\mu \rightarrow e \gamma$, their current experimental bounds 

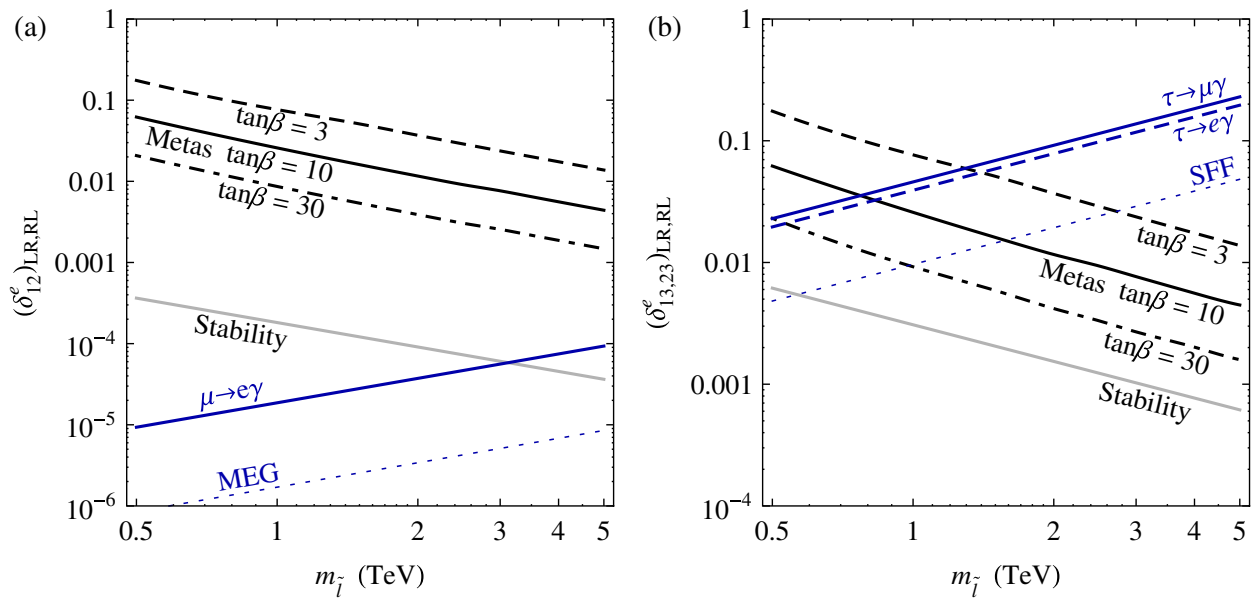

FIG. 6 (color online). Comparison of constraints on $\left(\delta_{i j}^{e}\right)_{L R}$. The thick black lines with negative slopes are bounds from the requirement that $S>400$ for three values of $\tan \beta$ represented by different dashing styles. The thick grey lines are the stability bounds. The thick dark grey (blue) lines with positive slopes are the current bounds from flavor-changing processes. The thin dotted (blue) lines are anticipated experimental bounds.

are less powerful and leave ample room for vacuum metastability even for slepton masses as low as $500 \mathrm{GeV}$. If one were to insist on permanent stability, it could be an explanation for why LFV in $\tau$ decays has not been discovered yet. This requirement would rule out even the possibility of observing $\tau \rightarrow e \gamma$ or $\tau \rightarrow \mu \gamma$ at a SFF, provided that they arise solely from the trilinear terms and that $m_{\tilde{l}} \gtrsim 600 \mathrm{GeV}$. Adopting the vacuum longevity constraint instead makes the LFV search more promising. A SFF might be able to find the virtual effect of a $L R$ insertion involving the third family if sleptons are lighter than 1.0, 1.6, $2.7 \mathrm{TeV}$ for $\tan \beta=30$, 10,3 , respectively. If one wishes to use the projected $\tau \rightarrow \mu \gamma$ bound of $3 \times 10^{-9}$ from Ref. [30], one may shift up the corresponding line in the plot by the factor of $\sqrt{3 / 2}$.
Next, the down-type quark sector is investigated. The structure of each of Figs. 7 is the same as a leptonic one. The left panel is devoted to the $s \rightarrow d$ transition. The kaon physics constraint is weaker than the stability condition but stronger than metastability up to the mass scale around a few TeV. Again, the crossing position varies according to $\tan \beta$ which can be found in Fig. 7(a). If squarks are heavier than this mass scale, $\left(\delta_{12}^{d}\right)_{L R, R L}$ are bounded to be smaller than the sensitivity of present measurements. However, one should keep in mind that kaon dynamics is plagued by large theoretical uncertainties and so is the experimental limit.

The mass insertions relevant to the bottom quark are shown in Fig. 7(b). The features of the $1-3$ and the 2-3
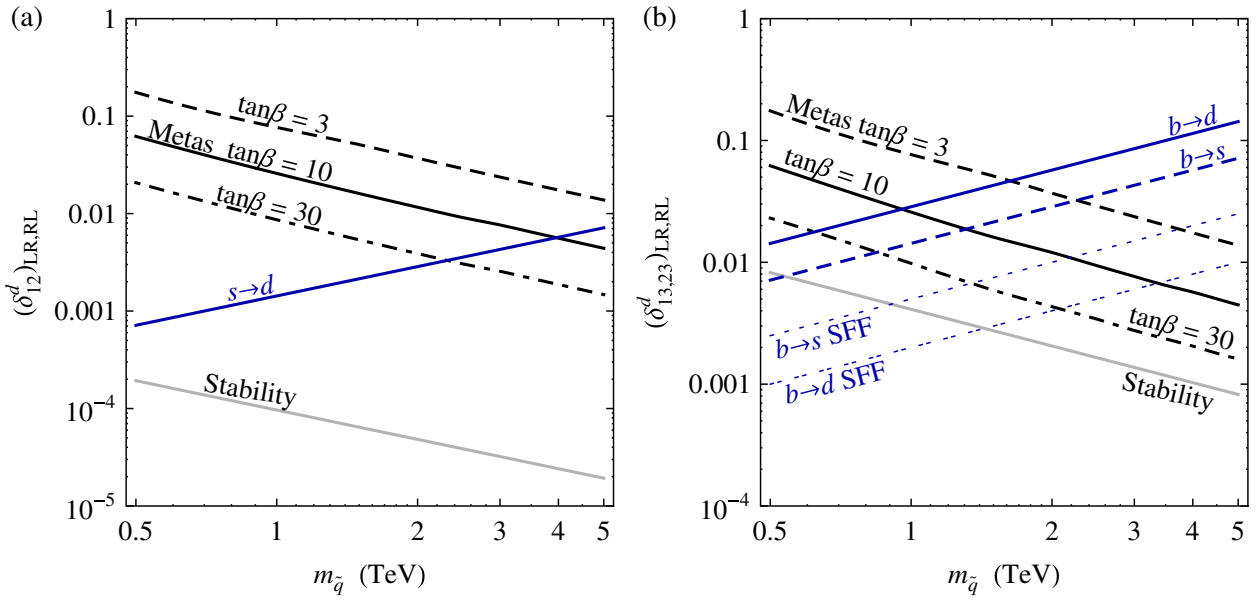

FIG. 7 (color online). Comparison of constraints on $\left(\delta_{i j}^{d}\right)_{L R}$. The thick black lines with negative slopes are bounds from the requirement that $S>400$ for three values of $\tan \beta$ represented by different dashing styles. The thick grey lines are the stability bounds. The thick dark grey (blue) lines with positive slopes are the current bounds from flavor-changing processes. The thin dotted (blue) lines are anticipated experimental bounds. 
sectors are similar, with differences arising from the FCNC constraints. The current $B$ physics data does not give much information with respect to whether or not a vacuum decay can be triggered by the related trilinear term. As long as one stays within the stable region, the scope of a SFF stops around $1.4 \mathrm{TeV}$ via the 1-3 mixing and a little lower via $2-3$. If one goes beyond the stability limit, the reach of new physics search in $b \rightarrow d$ transitions is raised up to 2.1, 3.4, $5.9 \mathrm{TeV}$ for $\tan \beta=30,10,3$, respectively. The respective $b \rightarrow s$ counterparts are 1.3, 2.2, 3.7 TeV. As before, these limits are set by the age of the Universe. Remember that there are hadronic uncertainties in heavy flavor physics as well, albeit less than in the kaon sector. Also, the future bounds in Table III have rather big errors. In particular, new physics is much easier to unveil if there is an extra complex phase, since $C P$ violation is less affected by hadronic uncertainties in general. These points should be taken into account when one interprets the above numerical results.

Before moving on to the up sector, it should be appropriate to recall the Higgs mass dependence. The preceding graphs correspond to the choice $m_{H^{0}}=0.5 \mathrm{TeV}$. As demonstrated in Fig. 4(a), higher $m_{H^{0}}$ makes bigger $\left(\delta_{i j}^{e, d}\right)_{L R}$ acceptable. This can extend the boundary of reachable mass upwards.

Finally, Fig. 8 is allocated to the flavor violation of the up-type squarks. One finds an outstanding distinction between the metastability bounds shown here and in the preceding plots, i.e. the way they depend on $\tan \beta$. This is because the up-type squarks couple to $H_{u}$ unlike the fields considered above. According to (6), the bound becomes looser as $\tan \beta$ increases although the dependence is much milder.

Regarding the 1-2 sector, there is a constraint from a flavor-changing process. In Fig. 8(a), the $D^{0}-\bar{D}^{0}$ mixing

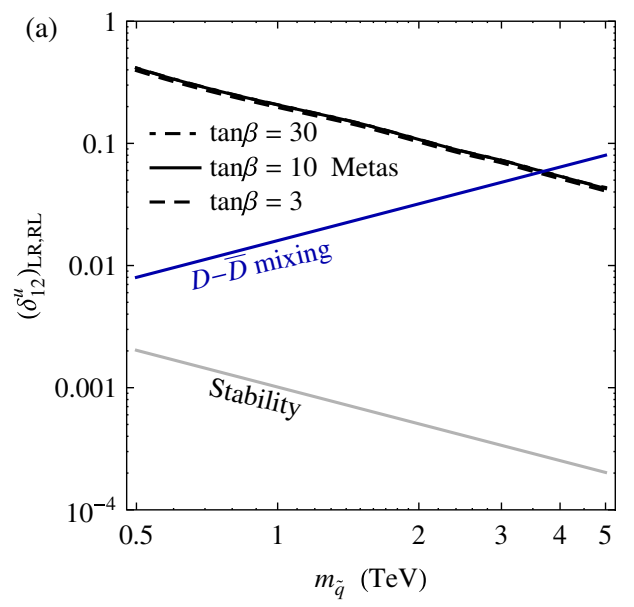

bound is plotted. It does not forbid the SML vacuum having a finite lifetime, but does restrict the tunnelling rate below the acceptable level for $m_{\tilde{q}} \lesssim 4 \mathrm{TeV}$. Beyond this mass scale, the metastability bound is stricter than the current data.

There is not much experimental information on the $L R$ insertions related to the top squark. In particular, no FCNC constraint is available yet. Instead, Fig. 8(b) employs another indirect limit coming from electroweak precision observables. It is obtained by requiring that the squark contribution to the $\rho$ parameter [35] does not exceed $5.5 \times 10^{-4}$ [36]. It is based on the assumption that $\left(\delta_{i j}^{d}\right)_{L R}$ is not correlated with $\left(\delta_{i j}^{u}\right)_{L R}$ so that a large value of the latter breaks the custodial symmetry between the upand down-type squarks significantly. As squarks grow heavier, this limit does not decouple either but tightens. However, the pace is slower than those of the vacuum constraints. As a result, the vacuum longevity bound is more stringent in most of the parameter space. Another point to notice is that the stability and the metastability curves are very close to each other compared to the foregoing cases that do not involve the top quark. This is mainly because the large top Yukawa coupling renders the inequality (10) less restrictive. In the plot is shown even a small region which is ruled out by the tunnelling constraint for some $\tan \beta$ even though it satisfies the stability requirement. Of course, a classically stable vacuum cannot decay. This apparent nonsense stems from the fact that the condition (10), used for drawing the curve, is not optimal [21]. That is, obeying this condition is not enough to exclude any $\mathrm{CCB}$ minimum to which tunnelling can occur. It is beyond the scope of this article to pin down the necessary and sufficient conditions for vacuum stability with respect to the flavor-violating trilinear terms. In any case, the stability bound is not the primary concern here.

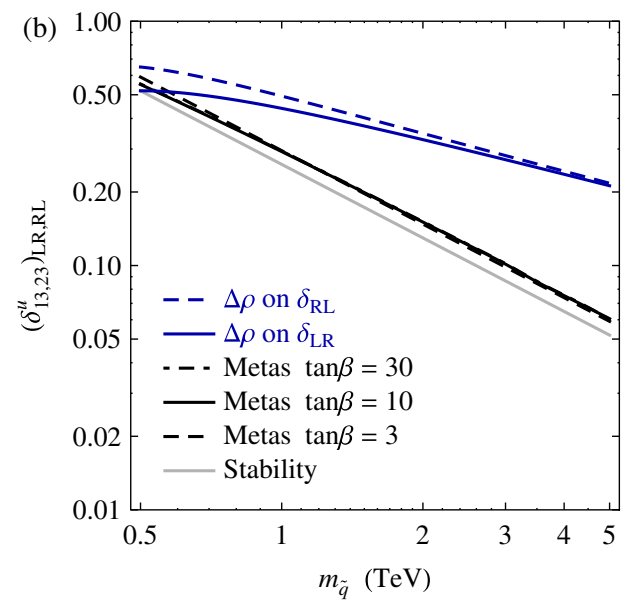

FIG. 8 (color online). Comparison of constraints on $\left(\delta_{i j}^{u}\right)_{L R}$. The thick black lines are bounds from the requirement that $S>400$, for three values of $\tan \beta$ represented by different dashing styles. The thick grey lines are the stability bounds. The thick dark grey (blue) line in panel (a) is the current bound from $D^{0}-\bar{D}^{0}$ mixing. In panel (b), the thick dark grey (blue) lines are the bounds from the $\rho$ parameter. 
TABLE IV. Metastability and naturalness bounds for $m_{\tilde{q}}=$ $M_{3}=1 \mathrm{TeV}$. The metastability bounds on $\left(\delta_{i j}^{d}\right)_{L R}$ are proportional to $\cos \beta$ and the displayed values are for $\tan \beta=10$.

\begin{tabular}{lcc}
\hline \hline MI & Metastability & Naturalness \\
\hline$\left(\delta_{12}^{d}\right)_{L R}$ & 0.026 & 0.0011 \\
$\left(\delta_{13}^{d}\right)_{L R}$ & 0.026 & 0.0010 \\
$\left(\delta_{23}^{d}\right)_{L R}$ & 0.026 & 0.010 \\
$\left(\delta_{12}^{u}\right)_{L R}$ & 0.21 & 0.011 \\
$\left(\delta_{13}^{u}\right)_{L R}$ & 0.29 & 0.062 \\
$\left(\delta_{23}^{u}\right)_{L R}$ & 0.29 & 0.59 \\
\hline \hline
\end{tabular}

There is another class of theoretical bounds that scale as the inverse power of the sparticle masses. They are naturalness bounds that act on half of the flavor-changing squark mass insertions, $\left(\delta_{i j}^{d, u}\right)_{L R}$ with $i<j$ [37]. They are based on the requirement that the supersymmetric loop corrections do not exceed the measured values of the CKM matrix elements. They are compared with the tunnelling limits in Table IV. One can notice that metastability is tighter on $\left(\delta_{23}^{u}\right)_{L R}$ and that the two types of constraints are comparable on $\left(\delta_{23}^{d}\right)_{L R}$ especially for high $\tan \beta$. For the remaining four insertions, naturalness tends to be stronger. This strength depends on the degree of fine-tuning that one is willing to allow, which by contrast has nothing to do with the metastability bounds. Note that the slepton sector and the other half of the squark sector $L R$ insertions are not restricted by the naturalness in the renormalization of the lepton or the quark mixing matrix. These insertions can be constrained by two-loop corrections to light fermion masses but only in a combination with another insertion [38]. These multiple insertion bounds shall be left out of the present comparison as the vacuum lifetime limits a $L R$ insertion even if it is the only nonvanishing component.

\section{CONCLUSIONS}

In the context of the MSSM, upper bounds on the flavorviolating trilinear soft terms have been obtained by demanding that the standard vacuum be long-lived. Obviously, metastability leaves more room than stability. As with the latter, however, the former results in a limit that does not decouple even if $m_{\tilde{f}}$ increases. A distinct property of the new bounds is that those on the 12 components $A_{i j}^{e, d}$ are nearly the same. This is because the bounce almost follows a $D$-flat direction and the corresponding Yukawa couplings are negligible. Dependence on other parameters has been reported as well such as Higgs masses, $\tan \beta$, and $\mu$. The bounds are fairly stable against radiative corrections in view of the estimated errors.

Prospects for an indirect new physics discovery have been discussed in a scenario where flavor-violating trilinear terms can be arbitrarily large within the limits from vacuum longevity. Being sensitive to mass ranges as high as $60 \mathrm{TeV}$ or more, $\mu \rightarrow e \gamma$ could be the most powerful probe of new physics. The potential of a SFF has also been considered. It should be able to cover up to a few TeV through $B$ physics and LFV $\tau$ decays. Lower $\tan \beta$ tends to extend the territory, but it does not seem to grow an order-of-magnitude beyond the reach of the LHC. It should be reminded that these conclusions are based on the assumption that the $L R$ insertions are the only source of extra flavor violation. As for the stop couplings, metastability provides bounds that are stronger than any existing experimental constraints even for rather light squarks.

\section{ACKNOWLEDGMENTS}

The author thanks A. Ali, S. Baek, A. Crivellin, K. Fujikawa, M. Hazumi, N. Kevlishvili, O. Lebedev, and H. Nakano, for inspirational questions and comments.
[1] G. L. Kane, P. Ko, H. b. Wang, C. Kolda, J.-h. Park, and L. T. Wang, Phys. Rev. D 70, 03505 (2004); Phys. Rev. Lett. 90, 141803 (2003).

[2] F. Gabbiani, E. Gabrielli, A. Masiero, and L. Silvestrini, Nucl. Phys. B477, 321 (1996).

[3] J. M. Frere, D. R. T. Jones, and S. Raby, Nucl. Phys. B222, 11 (1983); L. Alvarez-Gaume, J. Polchinski, and M. B. Wise, Nucl. Phys. B221, 495 (1983); J. P. Derendinger and C. A. Savoy, Nucl. Phys. B237, 307 (1984); C. Kounnas, A. B. Lahanas, D. V. Nanopoulos, and M. Quiros, Nucl. Phys. B236, 438 (1984).

[4] J. A. Casas and S. Dimopoulos, Phys. Lett. B 387, 107 (1996).

[5] M. Bona et al., INFN Pisa, Italy, 2007, (INFN Publishing Services, Pisa, 2007).
[6] M. Quiros, Helv. Phys. Acta 67, 451 (1994).

[7] A. Kusenko, P. Langacker, and G. Segre, Phys. Rev. D 54, 5824 (1996).

[8] M. S. Carena, M. Quiros, and C. E. M. Wagner, Phys. Lett. B 380, 81 (1996); M. Carena, G. Nardini, M. Quiros, and C. E. M. Wagner, Nucl. Phys. B812, 243 (2009).

[9] M. Dine, L. Randall, and S. D. Thomas, Phys. Rev. Lett. 75, 398 (1995); J. R. Ellis, J. Giedt, O. Lebedev, K. Olive, and M. Srednicki, Phys. Rev. D 78, 075006 (2008).

[10] M. Claudson, L. J. Hall, and I. Hinchliffe, Nucl. Phys. B228, 501 (1983).

[11] M. Endo, K. Hamaguchi, and K. Nakaji, J. High Energy Phys. 11 (2010) 004; J. Hisano and S. Sugiyama, Phys. Lett. B 696, 92 (2011).

[12] A. Riotto and E. Roulet, Phys. Lett. B 377, 60 (1996). 
[13] A. Strumia, Nucl. Phys. B482, 24 (1996).

[14] J. L. Evans, D. E. Morrissey, and J. D. Wells, Phys. Rev. D 80, 095011 (2009).

[15] J. Hisano, M. Nagai, M. Senami, and S. Sugiyama, Phys. Lett. B 659, 361 (2008); J. Hisano, M. Nagai, S. Sugiyama, and T. T. Yanagida, Phys. Lett. B 665, 237 (2008).

[16] L. J. Hall, V. A. Kostelecky, and S. Raby, Nucl. Phys. B267, 415 (1986).

[17] S. R. Coleman, Phys. Rev. D 15, 2929 (1977); 16, 1248(E) (1977); C. G. Callan, Jr. and S. R. Coleman, Phys. Rev. D 16, 1762 (1977).

[18] S. R. Coleman, V. Glaser, and A. Martin, Commun. Math. Phys. 58, 211 (1978).

[19] S. P. Martin, Phys. Rev. D 65, 116003 (2002); 66, 096001 (2002).

[20] E. J. Weinberg, Phys. Rev. D 47, 4614 (1993).

[21] J. A. Casas, A. Lleyda, and C. Munoz, Nucl. Phys. B471, 3 (1996).

[22] T. Konstandin and S. J. Huber, J. Cosmol. Astropart. Phys. 06 (2006) 021.

[23] J.-h. Park, J. Cosmol. Astropart. Phys. 02 (2011) 023.

[24] A. Wächter and L. T. Biegler, Math. Program. 106, 25 (2005); the package and documentation are available at https://projects.coin-or.org/Ipopt.

[25] B. C. Allanach, Comput. Phys. Commun. 143, 305 (2002).
[26] S.P. Martin, in Advanced Series on Directions in High Energy Physics, edited by G. L. Kane (World Scientific, Singapore, 1998), Vol. 18.

[27] M.L. Brooks et al. (MEGA Collaboration), Phys. Rev. Lett. 83, 1521 (1999).

[28] A. Baldini et al., Research Proposal to INFN, September 2002, http://meg.web.psi.ch/.

[29] B. Aubert et al. (BABAR Collaboration), Phys. Rev. Lett. 104, 021802 (2010).

[30] T. Aushev et al., arXiv:1002.5012.

[31] M. Ciuchini, A. Masiero, P. Paradisi, L. Silvestrini, S. K. Vempati, and O. Vives, Nucl. Phys. B783, 112 (2007).

[32] M. Artuso et al., Eur. Phys. J. C 57, 309 (2008).

[33] P. Ko, J.-h. Park, and G. Kramer, Eur. Phys. J. C 25, 615 (2002).

[34] A. Crivellin and U. Nierste, Phys. Rev. D 81, 095007 (2010).

[35] S. Heinemeyer, W. Hollik, F. Merz, and S. Penaranda, Eur. Phys. J. C 37, 481 (2004).

[36] J. J. Cao, G. Eilam, M. Frank, K. Hikasa, G. L. Liu, I. Turan, and J.M. Yang, Phys. Rev. D 75, 075021 (2007).

[37] A. Crivellin and U. Nierste, Phys. Rev. D 79, 035018 (2009).

[38] A. Crivellin and J. Girrbach, Phys. Rev. D 81, 076001 (2010). 